\title{
L'INTERVENTO NUTRIZIONALE NEI DISTURBI DEL COMPORTAMENTO ALIMENTARE
}

\author{
MARIA LETIZIA PETRONI (*)
}

\begin{abstract}
SunTO. - L'intervento nutrizionale rappresenta uno dei cardini del trattamento dei disturbi del comportamento alimentare. Nei casi di maggiore gravità l'intervento iniziale è rappresentato dalla nutrizione artificiale, da condurre con cautela per prevenire la sindrome da re-alimentazione che può avere conseguenze anche fatali. Una volta stabilizzate le condizioni del paziente può iniziare l'intervento integrato: medico, nutrizionale, psicologico, motorio, educativo che può svolgersi inizialmente in un setting ospedaliero e successivamente in un setting ambulatoriale. La rieducazione nutrizionale nell'anoressia nervosa può essere condotta con la modalità della alimentazione meccanica o con quella della desensibilizzazione dell'ansia da cibo. Obiettivo è la progressiva normalizzazione del peso e dell'alimentazione e il miglioramento della qualità della vita del paziente.
\end{abstract}

$$
* * *
$$

ABSTRACT. - The nutritional intervention is a cornerstone of the treatment of eating disorders. Most serious cases are being treated first by parenteral and/or enteral nutrition, carefully to prevent the re-feeding syndrome that can have fatal consequences. Once patient is clinically stable, integrated intervention can be started: medical, nutritional, psychological, motor, rehabilitation and therapeutic education may take place initially in a hospital setting and then in an outpatient setting. The nutritional rehabilitation in anorexia nervosa can be conducted with the mode of the mechanical feeding or with that of desensitization of anxiety by food. Goal is the gradual normalization of weight and nutrition and improving the quality of life of the patient.

La riabilitazione nutrizionale nei pazienti con disturbi del comportamento alimentare ha come obiettivi il ripristino durevole di corrette abitudini alimentari (qualità, quantità, ritmo) e della normalizza-

(*) Centro Studi Obesità, Forlì, Italia. E-mail: marialetizia.petroni@gmail.com 
zione della composizione corporea (massa magra, massa grassa, densità minerale ossea, acqua ed elettroliti). L'intervento nutrizionale deve essere sempre affrontato in un'ottica educativa e nell'ambito di un progetto interdisciplinare che coinvolga vari professionisti e - ove possibile- la famiglia. Nei casi di malnutrizione grave o comunque di elevato rischio per la vita del paziente l'intervento iniziale viene condotto in ambito ospedaliero - possibilmente presso nosocomi con le adeguate competenze - utilizzando nutrizione artificiale (per via endovenosa o tramite sonda naso gastrica) (2). Raramente può rendersi necessario il ricorso a un trattamento sanitario obbligatorio. Cautela deve essere posta nella prevenzione della cosiddetta sindrome da renutrizione che può colpire pazienti gravemente malnutriti e rialimentati troppo precocemente ed in maniera eccessiva, come avvenuto con esiti infausti al termine della Seconda Guerra Mondiale ai prigionieri liberati dai campi di sterminio nazisti. Una volta stabilizzate le condizioni del paziente può essere iniziato il trattamento riabilitativo vero e proprio che associa intervento nutrizionale, intervento psicologico, rieducazione funzionale e ricondizionamento fisico, educazione terapeutica, nursing riabilitativo, terapia farmacologica.

L'intervento riabilitativo nutrizionale nell'anoressia nervosa può essere condotto attraverso due principali modalità: i) la alimentazione meccanica, nella quale viene data priorità al ripristino in tempi brevi di una normale quota calorica assunta per os come condizione necessaria per la normale sintesi di neurotrasmettitori e la riduzione di alcuni comportamenti ossessivi perpetuanti il disturbo alimentare; ii) la desensibilizzazione dell'ansia da cibo, che viene condotta attraverso un graduale aumento delle ingesta a partire dagli alimenti meno ansiogeni per il paziente per arrivare progressivamente alla reintroduzione di alimenti fortemente ansiogeni in quanto ritenuti a torto od a ragione "ingrassanti" da parte del paziente. La scelta della modalità più opportuna è in funzione di diversi fattori, alcuni dipendenti dalle caratteristiche (psicologiche e non) del paziente, altri dalla esperienza degli operatori e dall'organizzazione della struttura riabilitativa. Con un intervento di nutrizione intensiva (se accettato dal paziente) che parta da $30-40 \mathrm{kcal} / \mathrm{kg}$ peso per arrivare a $70-100 \mathrm{kcal} / \mathrm{kg}$ peso è possibile arrivare ad un aumento ponderale sino a $1-1,5 \mathrm{~kg} / \mathrm{settimana}(1)$.

Gli obiettivi di progressiva normalizzazione del peso e dell'alimentazione e di miglioramento della qualità della vita vengono condivisi con il paziente, che grazie all'intervento integrato multidisciplinare viene aiu- 
tato a tollerare l'ansia creata dall'aumento ponderale e a ridurre pensieri e comportamenti disfunzionali. Il dietista - tecnico della riabilitazione nutrizionale - rappresenta la figura chiave nel trattamento riabilitativo del paziente (2). Oltre ai colloqui individuali e all'educazione alimentare di gruppo il dietista svolge tra le sue attività il pasto assistito, il supporto alla preparazione del pasto da parte dei pazienti nella cucinetta di reparto; nella fase di preparazione al rientro al domicilio accompagna $\mathrm{i}$ pazienti in uscite guidate presso supermercati e ristoranti.

Secondo la Consensus SISDCA (3), il programma di trattamento del paziente con DCA in riabilitazione intensiva deve sempre integrare, in un approccio interdisciplinare:

1) intervento nutrizionale finalizzato a:

a. ottenere un graduale recupero ponderale con un buon equilibrio massa grassa/massa magra;

b. ricostruire durevolmente corrette abitudini alimentari (qualità, quantità, ritmo) basate sui canoni della Dieta Mediterranea anche in una logica educazionale;

c. ottenere una compliance del paziente funzionale al raggiungimento degli obiettivi.

2) programma di rieducazione funzionale e ricondizionamento fisico, finalizzato a:

a. riattivare strutture muscolari ipotoniche e ipotrofiche insegnando esercizi muscolari adeguati;

b. migliorare la performance cardio-circolatoria e respiratoria;

c. bilanciare la spesa energetica con l'introito calorico e le necessità nutrizionali;

d. migliorare il rapporto massa magra/massa grassa;

e. favorire una più corretta distribuzione della massa grassa nella fase di recupero ponderale;

f. contrastare il depauperamento della densità minerale ossea;

g. rieducare il paziente a uno stile di vita attivo e salutare in alternativa all'attività fisica compulsiva spesso presente in alcuni DCA.

3) educazione terapeutica: interventi psico-pedagogici e psicoterapeutici brevi focalizzati, condotti da operatori abilitati e formati, diretti in particolare a:

a. riconoscere i reali bisogni dei pazienti;

b. correggere le convinzioni errate dei pazienti sull'alimentazione e l'attività fisica che talvolta rappresentano il primo vero ostacolo alla cura; 
c. migliorare non solo le conoscenze, ma anche le competenze dei pazienti attraverso il passaggio dal "sapere" al "saper fare" e "saper essere";

d. facilitare non solo l'apprendimento cognitivo (attraverso la condivisione di argomenti specifici) ma anche l'apprendimento esperienziale (grazie all'uso di role playing e simulate) e quello intuitivo (attraverso l'uso di aforismi, metafore, storie);

e. insegnare il controllo di semplici parametri clinici (frequenza cardiaca, glicemia nei diabetici);

f. allenare alla gestione e all'autocontrollo dell'alimentazione, dell'attività fisica, dei momenti di stress ed ansia (diario alimentare, automonitoraggio, controllo degli stimoli, problem solving);

g. contrastare le pratiche improprie e dannose di controllo del peso corporeo (vomito, lassativi etc.);

h. migliorare il rapporto con il corpo e la sua immagine (danzamovimento-terapia, training autogeno e altre tecniche corporee di rilassamento);

i. aumentare il senso di responsabilità nella malattia e nella cura (illness behaviour);

j. favorire la compliance terapeutica (intervista motivazionale, strategie di counseling motivazionale, ...).

4) nursing riabilitativo (rebabilitation nursing): interventi svolti da infermieri e diretti a:

- migliorare le risposte dei pazienti a malattie croniche, disabilità e stili di vita patogeni;

- potenziare i supporti ed i compensi ambientali e sociali;

- proteggere e stimolare le capacità funzionali e relazionali al fine di migliorare la partecipazione alle attività riabilitative ed ai programmi assistenziali.

5) terapia farmacologica in casi selezionati;

6) psicoterapia, in particolare di gruppo, motivazionale, breve e focalizzata.

Gli interventi psicoterapeutici maggiormente strutturati devono essere riservati, in linea di massima, al setting ambulatoriale, ma vanno preparati e predisposti durante i ricoveri riabilitativi in funzione di un adeguato piano terapeutico post-dimissione.

L'intervento riabilitativo nutrizionale nella bulimia nervosa viene condotto con lo scopo di aiutare il paziente ad uscire dal "caos alimentare" rappresentato dal circolo vizioso abbuffata-vomito (od iperattività 
fisica od abuso di lassativi o diuretici). Il paziente viene aiutato alla percezione dei fisiologici segnali di fame e sazietà ed alla ripresa di normali ritmi alimentari. L'utilizzo di strumenti di misurazione del metabolismo basale (calorimetria indiretta) o della composizione corporea (bioimpedenziometria, plicometria) facilita l'adesione del paziente alla terapia nutrizionale e cognitivo-comportamentale.

L'intervento riabilitativo nutrizionale nel disturbo da alimentazione incontrollata associato all'obesità rispecchia per alcune modalità quello per la bulimia nervosa (4). Particolare attenzione deve essere posta alla prevenzione della sindrome dell'oscillazione del peso che è il risultato di diete drastiche con rapide e massive riduzioni di peso poi seguite da un altrettanto rapido recupero, spesso a livelli superiori stante la riduzione della massa magra metabolicamente attiva. Importante anche aiutare il paziente a convivere con le proprie forme corporee stante la forte componente genetica dell'obesità - e quindi a raggiungere e mantenere nel tempo il proprio "peso ragionevole".

\section{BIBLIOGRAFIA ESSENZIALE}

1) Marzola E, Nasser JA, Hashim SA, Shih PA, Kaye WH. Nutritional rehabilitation in anorexia nervosa: review of the literature and implications for treatment. BMC Psychiatry. 2013;13:290. doi: 10.1186/1471-244X-13-290.

2) American Dietetic Association. Position of the American Dietetic Association: Nutrition intervention in the treatment of anorexia nervosa, bulimia nervosa, and other eating disorders. J Am Diet Assoc. 2006;106:2073-82.

3) Donini LM, Cuzzolaro M, Spera G, et al. [Obesity and Eating Disorders. Indications for the different levels of care. An Italian Expert Consensus Document].Eat Weight Disord. 2010;15 (1-2 Suppl):1-31.

4) Amianto F, Ottone L, Abbate Daga G, Fassino S. Binge-eating disorder diagnosis and treatment: a recap in front of DSM-5. BMC Psychiatry. 2015 Apr 3;15:70. doi: 10.1186/s12888-015-0445-6. 
